\title{
Presenting parabolic subgroups
}

\author{
FRANÇOIS DAHMANI \\ VINCENT GUIRARDEL
}

\begin{abstract}
Consider a relatively hyperbolic group $G$. We prove that if $G$ is finitely presented, so are its parabolic subgroups. Moreover, a presentation of the parabolic subgroups can be found algorithmically from a presentation of $G$, a solution of its word problem and generating sets of the parabolic subgroups. We also give an algorithm that finds parabolic subgroups in a given recursively enumerable class of groups.
\end{abstract}

20F67; 20F10

Consider a relatively hyperbolic group $G$ with parabolic subgroups $H_{1}, \ldots, H_{n}$. It is well known that if each $H_{i}$ is finitely generated (or finitely presented), then so is $G$. Osin showed conversely that if $G$ is finitely generated, then so are $H_{1}, \ldots, H_{n}$ [9, Proposition 2.27]. Whether finite presentation of $G$ implies finite presentation of $H_{1}, \ldots, H_{n}$ is an important question raised by Osin in [9, Problem 5.1].

On the algorithmic side, given a finite presentation of a relatively hyperbolic group $G$ and a generating set of the parabolic subgroups, can one find a presentation of the parabolic subgroups?

We give a positive answer to these two questions.

Theorem 1 Let $G$ be a finitely presented group. Assume that $G$ is hyperbolic relative to $H_{1}, \ldots, H_{n}$. Then each $H_{i}$ is finitely presented.

Theorem 2 There exists an algorithm that takes as input a finite presentation of a group $G$, a solution to its word problem and a collection of finite subsets $S_{1}, \ldots, S_{n} \subset G$, and that terminates if and only if $G$ is hyperbolic relative to $\left\langle S_{1}\right\rangle, \ldots,\left\langle S_{n}\right\rangle$.

In this case, the algorithm outputs a linear isoperimetry constant $K$ for the corresponding relative presentation, a finite presentation for each of the parabolic subgroups $\left\langle S_{i}\right\rangle$, and says whether $G$ is properly relative hyperbolic relative to $\left\langle S_{1}\right\rangle, \ldots,\left\langle S_{n}\right\rangle$ (ie $\left\langle S_{i}\right\rangle \varsubsetneqq G$ for all $\left.i\right)$.

In this statement, the linear isoperimetry constant $K$ is for the relative presentation $X_{\infty}$ as defined in Section 1.2.

If one is not given generating sets of the parabolic subgroups, one can search for them, and require that they lie in some recursively enumerable class of groups. 
Theorem 3 There exists an algorithm as follows. It takes as input a finite presentation of a group $G$, a solution for its word problem and a recursive class of finitely presented groups $\mathcal{C}$ (given by a Turing machine enumerating presentations of these groups).

It terminates if and only if $G$ is properly hyperbolic relative to subgroups that are in the class $\mathcal{C}$.

In this case, the algorithm outputs an isoperimetry constant $K$, a generating set and a finite presentation for each of the parabolic subgroups.

The Turing machine enumerating $\mathcal{C}$ is a machine that enumerates some finite presentations, each of which represents a group in $\mathcal{C}$, and such that every group in $\mathcal{C}$ has at least one presentation that is enumerated.

This paper can be seen as a continuation, extension and precision on the form and the substance of the first author's [3]. It is based on the analysis of some van Kampen diagrams in different truncated relative presentations. The main tool is Proposition 2.9, which says that if some relative presentation does not satisfy a linear isoperimetric inequality, then this shows up on some diagram of small area and small complexity.

Section 1 recalls definitions about isometric inequalities, introduces truncated relative presentations, and defines the complexity of a diagram. Section 2 contains the main technical results. Section 3 is devoted to corollaries. Theorems 1, 2 and 3 follow from Corollaries 3.3, 3.5 and 3.6.

Acknowledgement Both authors are supported by the funding of the project ANR11-BS01-013.

\section{Context}

\subsection{Linear isoperimetric inequalities}

A presentation is a pair $(S \mid \mathcal{R})$, where $S$ is a set, and $\mathcal{R} \subset \mathbb{F}_{S}$ is a subset of the free group $\mathbb{F}_{S}$. The group defined by this presentation is $\left.\langle S \mid \mathcal{R}\rangle=\mathbb{F}_{S} /\langle\mathcal{R}\rangle\right\rangle$. If $G$ is a given group and $\sigma: S \rightarrow G$ is a map from a set $S$ to $G$, we say that $(S \mid \mathcal{R})$ is a presentation of $G$ (with respect to the map $\sigma$ ) if $\sigma(S)$ generates $G$ and $\mathcal{R}$ normally generates the kernel of the natural map $\mathbb{F}_{S} \rightarrow G$, ie if $\sigma$ extends to an isomorphism $\langle S \mid \mathcal{R}\rangle \rightarrow G$. The elements of $\mathcal{R}$ are called defining relations, and we usually write $G=\langle S \mid \mathcal{R}\rangle$.

Note that we use distinct notations for the group and its presentation, we will usually denote by $X=(S \mid \mathcal{R})$ the presentation, and by $G=\langle S \mid \mathcal{R}\rangle$ the group defined by this presentation. 
We denote by $\bar{s}$ the inverse of the basis element $s \in \mathbb{F}_{S}$ and we view an element of $\mathbb{F}_{S}$ as a reduced word over the alphabet $S \cup \bar{S}$. We say that a presentation is triangular if every defining relation has length at most 3 (as word over $S \cup \bar{S}$ ). If one allows to increase the generating set, it is not restrictive to consider triangular presentations: from an arbitrary finite presentation, one can effectively construct a triangular one.

If a word $w \in \mathbb{F}_{S}$ represents the trivial element of $G$ (we write $w \stackrel{G}{=} 1$ ), the area of $w$ for the presentation $X=(S \mid \mathcal{R})$, denoted by $\operatorname{Area}(w)$, is the minimal number $n$ such that $w$ is the product in $\mathbb{F}_{S}$ of $n$ conjugates of elements of $\mathcal{R} \cup \mathcal{R}^{-1}$.

Given a word $w \in \mathbb{F}_{S}$ such that $w \stackrel{G}{=} 1$, a van Kampen diagram for $w$ over the presentation $X=(S \mid \mathcal{R})$ is a simply connected planar 2-complex $D$ such that oriented edges are labeled by elements of $S \cup \bar{S}$, such that reversing the orientation changes the label to its inverse, such that every 2 -cell has its boundary labeled by a cyclically reduced word conjugate to an element of $\mathcal{R} \cup \mathcal{R}^{-1}$, and such that the topological boundary $\partial D$ of $D$ is labeled by $w$. Sometimes, we just say cell instead of $2-$ cell. It is well known that $\operatorname{Area}(w)$ is the minimal number of 2-cells of van Kampen diagrams for $w$. See Lyndon and Schupp [8, Section 5.1] for more details.

An isoperimetric function of a presentation $X=(S \mid \mathcal{R})$ is a function $f: \mathbb{N} \rightarrow \mathbb{N}$ such that for all $w \in \mathbb{F}_{S}$ representing the trivial element, $\operatorname{Area}(w) \leq f(\operatorname{length}(w))$. Note that if $S$ is infinite, there are infinitely many words of a given length, and it may happen that no such function (with finite values) exists.

Our approach is based on the fact that a group is relatively hyperbolic if and only if it has a presentation of a particular kind with a linear isoperimetric function [9]; see Theorem 1.2 below. Another important fact is that the failure of a specific linear isoperimetric inequality can be observed in a set of words of controlled area (Gromov [5], Bowditch [1] and Papasoglu [10]).

Theorem $1.1[10]$ Let $X=(S \mid \mathcal{R})$ be an arbitrary (not necessarily finite) triangular presentation of an arbitrary group $G$ and let $K \geq 1$.

Assume that there is a word $w \in \mathbb{F}_{S}$ such that $w \stackrel{G}{=} 1$ and $\operatorname{Area}(w)>K \operatorname{length}(w)$. Then there exists a word $w^{\prime} \in \mathbb{F}_{S}$ such that $w^{\prime} \underline{=} 1$ and such that:

- $\operatorname{Area}\left(w^{\prime}\right) \in\left[\frac{K}{2}, 240 K\right]$

- $\operatorname{Area}\left(w^{\prime}\right)>\frac{1}{2 \times 10^{4}} \operatorname{length}\left(w^{\prime}\right)^{2}$

As in [3], we use the fact that the statement and the proof of this theorem do not use that the presentation $X$ is finite. Indeed, the argument considers a word of minimal area 
such that $\operatorname{Area}(w)>K$ length $(w)$, and extracts from a minimal diagram a subdiagram of controlled area with $\operatorname{Area}\left(D^{\prime}\right)>\left(1 / 2 \times 10^{4}\right) \operatorname{length}\left(\partial D^{\prime}\right)^{2}$. Our constant $K$ is $K^{2}$ in [10], where Papasoglu's $K$ is assumed to be an integer in the statement, but only the inequality $K \geq 1$ is used in [10].

\subsection{Truncated and exact relative presentations}

Since finite generation of a relatively hyperbolic group implies finite generation of its maximal parabolic subgroups [9, Proposition 2.27], we always assume that relatively hyperbolic groups and their maximal parabolic subgroups are finitely generated.

Let us now define the multiplication table of a group $H$. This is the subset $\mathcal{T}(H)$ of the free group $\mathbb{F}_{H}$ consisting of all words on the alphabet $H \cup \bar{H}$ of length 1,2 or 3 that map to the trivial element in $H$ under the morphism $\varphi: \mathbb{F}_{H} \rightarrow H$ induced by the identity map $H \rightarrow H$. Note that given $a \in H$ and its inverse $a^{-1} \in H$, the basis element $a^{-1}$ of $\mathbb{F}_{H}$ is distinct from the inverse $\bar{a}$ in $\mathbb{F}_{H}$ of the basis element $a$. It is clear that the (usually infinite) presentation $(H \mid \mathcal{T}(H))$ is a presentation of $H$ : the identity map $H \rightarrow H$ induces an isomorphism $\langle H \mid \mathcal{T}(H)\rangle \rightarrow H$.

Let $G$ be a finitely presented group, and $H_{1}, \ldots, H_{n}$ be finitely generated subgroups of $G$. For each $i$, let $S_{i}$ be a finite symmetric generating set of $H_{i}$. Consider a finite triangular presentation $G=\langle S \mid \mathcal{R}\rangle$, where $S$ is a finite symmetric generating set of $G$ containing each $S_{i}$, and $\mathcal{R}$ is a finite set of triangular relations over $S$.

We are going to introduce a family of infinite presentations $X_{\rho}$, indexed by $\rho \in \mathbb{N} \cup \infty$. To make the definitions clearer, we first introduce the presentation $X_{\infty}$. Let $\widetilde{H}_{1}, \ldots, \widetilde{H}_{n}$ be some groups, isomorphic to $H_{i}$ under an isomorphism $p_{i}: \widetilde{H}_{i} \rightarrow H_{i}$. We denote by $\widetilde{S}_{i}=p_{i}^{-1}\left(S_{i}\right)$ the corresponding generating set of $\tilde{H}_{i}$. Consider the disjoint union

$$
\widehat{S}=S \sqcup \tilde{H}_{1} \sqcup \cdots \sqcup \tilde{H}_{n},
$$

and $\sigma: \hat{S} \rightarrow G$ the map whose restriction to $S$ is the inclusion and whose restriction to $\widetilde{H}_{i}$ is $p_{i}$. Since $S \subset \widehat{S}$, each relator in $\mathcal{R}$ can be viewed as an element of $\mathbb{F} \hat{S}$. To identify the generating set $\widetilde{S}_{i}$ of $\widetilde{H}_{i}$ with the corresponding subset of $S$, we consider for each $\widetilde{S} \in \widetilde{S}_{i}$ the two-letter relator $\widetilde{S}^{-1} p_{i}(\widetilde{S}) \in \mathbb{F} \widehat{S}$, where the first letter $\widetilde{S}^{-1}$ lies in $\widetilde{H}_{i}$ and the second letter $p_{i}(\widetilde{s})$ lies in $S$ (because $S_{i} \subset S$ ). We define the finite subset $\mathcal{R}^{\prime} \subset \mathbb{F} \hat{S}$ as the union of $\mathcal{R}$ with the set of all these two-letter relators. Finally, each element of the multiplication table $\mathcal{T}\left(\tilde{H}_{i}\right)$ is naturally a word of length at most 3 in $\mathbb{F} \hat{S}$. Thus, one can define the relative presentation $X_{\infty}$ as

$$
X_{\infty}=\left(\widehat{S} \mid \mathcal{R}^{\prime} \cup \mathcal{T}\left(\tilde{H}_{1}\right) \cup \cdots \cup \mathcal{T}\left(\tilde{H}_{n}\right)\right) .
$$


The triangular presentation $X_{\infty}$ is a (usually infinite) presentation of $G$, with respect to $\sigma$.

Indeed, $\sigma$ extends to a morphism $\varphi$ from the group $G^{\prime}$ defined by $X_{\infty}$ to $G$, and there is a morphism $\psi: G \rightarrow G^{\prime}$ induced by the inclusion $S \subset \widehat{S}$. Since $\varphi \circ \psi=\mathrm{id}_{G}$, and since $\psi$ is onto, $\varphi$ and $\psi$ are inverse of each other.

Theorem 1.2 [9, Theorem 1.7, Definition 2.29] $G$ is hyperbolic relative to the subgroups $H_{1}, \ldots, H_{n}$ if and only if the relative presentation $X_{\infty}$ satisfies a linear isoperimetric inequality.

The subgroups $H_{1}, \ldots, H_{n}$ of $G$ are called the maximal parabolic subgroups. Since there is no risk of confusion, we will simply call them parabolic subgroups.

Remark 1.3 Osin includes all words of any length in the multiplication table. One easily checks that this does not change the result.

To introduce the truncated relative presentations $X_{\rho}$, we fix $\rho \in \mathbb{N} \cup \infty$. We are first going to define some auxiliary groups $\widetilde{H}_{i}{ }^{\rho}$ with epimorphisms $p_{i}{ }^{\rho}: \widetilde{H}_{i}{ }^{\rho} \rightarrow H_{i}$. For each subgroup $H_{i}$, consider a copy ${\widetilde{S_{i}}}_{i}{ }^{\rho}$ of $S_{i}$. Let $\mathcal{R}_{\rho}\left(S_{i}\right)$ be the set of all words in the alphabet $\widetilde{S}_{i}{ }^{\rho} \cup{\widetilde{S_{i}}}^{\rho}$, of length $\leq \rho$, whose image as words on the alphabet $S_{i}^{ \pm 1}$ define trivial elements in $H_{i}$. Then we define $\widetilde{H}_{i}{ }^{\rho}=\left\langle\widetilde{S}_{i}{ }^{\rho} \mid \mathcal{R}_{\rho}\left(S_{i}\right)\right\rangle$, and denote by $p_{i}{ }^{\rho}: \widetilde{H}_{i}{ }^{\rho} \rightarrow H_{i}$ the obvious epimorphism.

Note that for $\rho=+\infty, p_{i}{ }^{\rho}$ is an isomorphism, and $\widetilde{S}_{i}{ }^{\rho}$ (resp. $\widetilde{H}_{i}{ }^{\rho}$ ) is the set that we denoted $\widetilde{S}_{i}$ (resp. $\widetilde{H}_{i}$ ) above. For $\rho<\infty, \widetilde{H}_{i}{ }^{\rho}$ is finitely presented.

The presentation $X_{\rho}$ is analogous to $X_{\infty}$, using $\tilde{H}_{i}^{\rho}$ instead of $\tilde{H}_{i}$. Let

$$
\widehat{S}_{\rho}=S \sqcup \tilde{H}_{1}^{\rho} \sqcup \cdots \sqcup \tilde{H}_{n}^{\rho},
$$

and consider $\mathcal{R}_{\rho}^{\prime} \subset \mathbb{F} \widehat{S}_{\rho}$ consisting of $\mathcal{R}$ together with the set of two-letter words of the form $\widetilde{S}^{-1} p_{i}{ }^{\rho}(\widetilde{s})$, where $s \in \widetilde{S}_{i}{ }^{\rho}$. Then, we define the truncated relative presentation $X_{\rho}$ as

$$
X_{\rho}=\left(\widehat{S}_{\rho} \mid \mathcal{R}_{\rho}^{\prime} \cup \mathcal{T}\left(\tilde{H}_{1}^{\rho}\right) \cup \cdots \cup \mathcal{T}\left(\tilde{H}_{n}^{\rho}\right)\right)
$$

As above, this triangular presentation is still a presentation of $G$, with respect to the map $\sigma_{\rho}: \widehat{S}_{\rho} \rightarrow G$ that is the identity on $S$ and restricts to $p_{i}{ }^{\rho}$ on $\widetilde{H}_{i}{ }^{\rho}$. Indeed $\sigma_{\rho}$ extends to a morphism $\varphi$ from the group $G^{\prime}$ defined by $X_{\rho}$ to $G$ and there is a morphism $\psi: G \rightarrow G^{\prime}$ induced by the inclusion $S \subset \widehat{S}$. Since $\varphi \circ \psi=\mathrm{id}_{G}$, and since $\psi$ is onto, $\varphi$ and $\psi$ are inverse to each other. 
We say that the truncated presentation $X_{\rho}$ is exact if for all $i, p_{i}{ }^{\rho}: \widetilde{H}_{i}{ }^{\rho} \rightarrow H_{i}$ is an isomorphism. By definition, $X_{\infty}$ is always exact. If $X_{\rho}$ is exact for some $\rho<\infty$, then all $H_{i}$ are finitely presented. Conversely, if all $H_{i}$ are finitely presented, then $X_{\rho}$ is exact for $\rho$ large enough (and $X_{\rho}$ is exactly the same presentation as $X_{\infty}$ ).

In Section 3, we are going to prove that if $X_{\infty}$ satisfies a linear isoperimetric inequality, so does $X_{\rho}$ for $\rho$ large enough. This will easily imply that parabolic subgroups are finitely presented.

\subsection{Complexities}

Since $X_{\rho}$ is an infinite presentation, it is convenient to have a measure of complexity for letters and words on $\hat{S}$. Recall that $\widehat{S}_{\rho}=S \sqcup \widetilde{H}_{1}{ }^{\rho} \sqcup \cdots \sqcup \widetilde{H}_{n}^{\rho}$. For $a \in \widetilde{H}_{i}{ }^{\rho}$, we denote by $|\widetilde{a}| \tilde{S}_{i}{ }^{\rho}$ the word length of $a$ relative to the generating set $\widetilde{S}_{i}{ }^{\rho}$. We define the complexity $\|a\|$ of $a \in \widehat{S}_{\rho}$ by $\|a\|=1$ if $a \in S$, and by $\|a\|=|a| \tilde{S}_{i}{ }^{\rho}$ if $a \in \widetilde{H}_{i}{ }^{\rho}$.

Given a word $w=a_{1} \cdots a_{n}$ over $\hat{S}_{\rho}$, we define:

- length $(w)=n$

- $\|w\|_{1}=\sum_{i=1}^{n}\left\|a_{i}\right\|$

- $\|w\|_{\infty}=\max _{i=1, \ldots, n}\left\|a_{i}\right\|$

Note that if $w$ is a one-letter word, then $\|w\|_{1}=\|w\|_{\infty}=\|w\|$.

Similarly, if $D$ is a diagram (or a path) whose edges are labeled by elements of $\widehat{S}_{\rho}$, we define $\|D\|_{1}$ and $\|D\|_{\infty}$ as the sum and the maximum of the complexities of the labels of its edges. For a labeled path $p$, length $(p)$ denotes its number of edges, and $\operatorname{Area}(D)$ denotes the number of $2-$ cells of a diagram $D$.

\section{Diagrams}

The goal of this section is to prove that if $X_{\rho}$ does not satisfy a linear isoperimetric inequality, this shows up on diagrams of small area and small complexity (Proposition 2.9).

\subsection{Vocabulary}

Thickness Let $D$ be a van Kampen diagram over the presentation $X_{\rho}$ ( $\rho$ being fixed in $\mathbb{N} \cup\{\infty\}$ ). We denote by $D_{\text {thick }} \subset D$ the union of all 2-cells, and of all vertices and edges that are contained in the boundary of a 2-cell. We say that $D$ is thick if $D=D_{\text {thick }}$ ie if every edge lies in the boundary of a 2-cell. 
Clusters We define cells of type $\mathcal{R}^{\prime}$ (resp. of type $\widetilde{H}_{i}{ }^{\rho}$ ) as those labeled by a word of $\mathcal{R}^{\prime}$ (resp. by a word in $\mathcal{T}\left(\widetilde{H}_{i}{ }^{\rho}\right)$ ). Any cell having an edge labeled by an element of $\widetilde{H}_{i}{ }^{\rho}$ is either a cell of type $\widetilde{H}_{i}{ }^{\rho}$, in which case its other edges are also labeled by elements of $\widetilde{H}_{i}{ }^{\rho}$, or is labeled by a two-letter word in $\mathcal{R}^{\prime} \backslash \mathcal{R}$, and its unique other edge is labeled by an element of $S$. Note in particular that two cells of type $\widetilde{H}_{i}{ }^{\rho}$ and $\widetilde{H}_{j}{ }^{\rho}$ cannot share an edge if $i \neq j$.

Two cells of the same type $\widetilde{H}_{i}{ }^{\rho}$ and sharing an edge are said to be cluster-adjacent. A cluster is an equivalence class for the transitive closure of this relation. All 2-cells of a cluster have the same type $\widetilde{H}_{i}{ }^{\rho}$, which we define as the type of the cluster. We identify a cluster with the closure $C$ of the 2 -cells it is made of. Note that clusters are contained in $D_{\text {thick }}$.

If $C$ is a cluster, we denote by $\partial C$ its topological boundary, ie the union of closed edges of $C$ that are in only one $2-$ cell of $C$.

Remark 2.1 Note that for any cluster $C$, any edge $e$ in $\partial C \backslash \partial D$ has complexity $\|e\|_{1}=\|e\|_{\infty} \leq 1$. Indeed, the $2-$ cell of $D \backslash C$ containing this edge is labeled by a relator $\widetilde{s}^{-1} p_{i}{ }^{\rho}(\widetilde{s})$ for some $\tilde{s} \in \widetilde{S}_{i}{ }^{\rho}$.

\subsection{Simply connected clusters, standard filling}

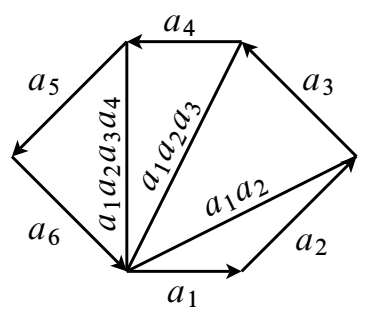

Figure 1: Standard filling

Note that a cluster $C$ (as a subset of the plane) is simply connected if and only if $C$ is a disk and $\partial C$ is an embedded circle in the plane. We will mostly deal with diagrams whose clusters are simply connected.

Consider a simply connected cluster $C$, with $\partial C$ labeled by the cyclic word $a_{1}, \ldots, a_{n}$ (where each $a_{j} \in \widetilde{H}_{i}{ }^{\rho} \cup{\widetilde{\tilde{H}_{i}}}^{\rho}$ ). If $n \geq 3$, a standard filling of $\partial C$ is a diagram with boundary $\partial C$, all whose vertices are in $\partial C$, and with $n-2$ triangles as in Figure 1. More precisely, if $v_{1}, \ldots, v_{n}$ are the vertices of $\partial C$, and its edges are $e_{1}, \ldots, e_{n}$ with $e_{j}$ joining $v_{j}$ to $v_{j+1}$ (modulo $n$ ) and labeled $a_{j} \in \widetilde{H}_{i}{ }^{\rho} \cup \overline{\widetilde{H}_{i}}{ }^{\rho}$, then for all $j=1, \ldots, n-2$, there is a triangle joining $v_{1}, v_{j+1}, v_{j+2}$, where the edge joining $v_{1}$ 
to $v_{j}$ is labeled by the image of the product $a_{1} \cdots a_{j}$ in $\tilde{H}_{i}{ }^{\rho}$. If $n \leq 2$, the standard filling of $\partial C$ is the diagram with boundary $\partial C$, and no other edge or vertex (it is a single cell that is a bigon or a monogon).

Lemma 2.2 If $C$ is an arbitrary simply connected cluster, then

$$
\|\partial C\|_{1} \leq 3 \operatorname{Area}(D)+\|\partial D\|_{1}
$$

If $C$ is standardly filled, then

$$
\operatorname{Area}(C)=\max \{1, \text { length }(\partial C)-2\} \quad \text { and } \quad\|C\|_{\infty} \leq\|\partial C\|_{1} .
$$

Proof Each edge of $\partial C \backslash \partial D$ has complexity at most 1 by Remark 2.1, and there are at most length $(\partial C) \leq 3 \operatorname{Area}(D)$ such edges. The sum of complexities of the edges in $\partial D$ is bounded by $\|\partial D\|_{1}$. This proves the first assertion. The second assertion is clear from the definition of a standard filling.

Remark 2.3 If $C$ is any cluster, then $\operatorname{Area}(C) \geq \operatorname{length}(\partial C)-2$. Indeed, denote by $F$ the number of 2-cells of $C$, and by $E_{\text {ext }}, E_{\text {int }}$, the number of edges in $\partial C$ and in $C \backslash \partial C$ respectively. Then, by connectedness of the dual graph, $F-1 \leq E_{\text {int }}$. Since cells of $C$ have at most 3 sides, $2 E_{\text {int }}+E_{\text {ext }} \leq 3 F$. It follows that $E_{\text {ext }} \leq F+2$ as required.

The following lemma shows that in many situations, clusters are simply connected.

Lemma 2.4 Let $w$ be a word over $\widehat{S}_{\rho}$ defining the trivial element in $G$. Let $D$ be a minimal van Kampen diagram for $w$ over the presentation $X_{\rho}$. Assume that $\rho \geq 3 \operatorname{Area}(D)$.

If $D$ is chosen among diagrams for $w$ over $X_{\rho}$ to minimize successively the area, and the number of 2-cells of type $\mathcal{R}^{\prime}$, then every cluster of $D$ is simply connected.

Assume either that $D$ is as above and that all its clusters are standardly filled, or that $D$ minimizes successively the area, the number of 2 -cells of type $\mathcal{R}^{\prime}$ and $\|D\|_{\infty}$. Then

$$
\|D\|_{\infty} \leq 3 \operatorname{Area}(D)+\|w\|_{1}
$$

Proof Assume for contradiction that there exists a cluster $C$ of type $\tilde{H}_{i}{ }^{\rho}$ that is not simply connected. Then there is a simply connected subdiagram $D^{\prime} \subset D$ such that edges of $\partial D^{\prime}$ are all in $\partial C \backslash \partial D$. Since edges of $\partial D^{\prime}$ lie in a $2-$ cell, length $\left(\partial D^{\prime}\right) \leq 3 \operatorname{Area}(D)$. Moreover $\left\|\partial D^{\prime}\right\|_{\infty}=1$, since by Remark 2.1, every edge in $\partial C \backslash \partial D$ has complexity 1. Thus, $\left\|\partial D^{\prime}\right\|_{1} \leq 3 \operatorname{Area}(D)$. Since $\rho \geq 3 \operatorname{Area}(D)$, the definition of $X_{\rho}$ says that 
the word labeled by $\partial D^{\prime}$ is trivial in $\widetilde{H}_{i}$. One can then replace the subdiagram bounded by $\partial D^{\prime}$ by a standardly filled diagram (with cells of type $\tilde{H}_{i}{ }^{\rho}$ ), that has smaller or equal area. This contradicts the minimality of $D$ for the number of 2 -cells of type $\mathcal{R}^{\prime}$. It follows that all clusters of $D$ are simply connected.

Assume now that all clusters are standardly filled. By Lemma 2.2, for each cluster $C,\|C\|_{\infty} \leq\|\partial C\|_{1} \leq 3 \operatorname{Area}(D)+\|w\|_{1}$. Since each edge of $D_{\text {thick }}$ of complexity at least 2 is contained in a cluster, this implies that $\left\|D_{\text {thick }}\right\|_{\infty} \leq 3 \operatorname{Area}(D)+\|w\|_{1}$.

Finally, assume that $D$ minimizes successively the area, the number of 2-cells of type $\mathcal{R}^{\prime}$ and $\|D\|_{\infty}$. Since clusters of $D$ are simply connected, we can modify $D$ to a diagram $D^{\prime}$ whose clusters are standardly filled, and having the same area and the same number 2-cells of type $\mathcal{R}^{\prime}$ as $D$. In particular, $\|D\|_{\infty} \leq\left\|D^{\prime}\right\|_{\infty}$. By the argument above, $\left\|D^{\prime}\right\|_{\infty} \leq 3 \operatorname{Area}(D)+\|w\|_{1}$ which concludes the proof.

\subsection{Complicated clusters}

A cluster $C$ is said to be complicated if $\partial C \cap \partial D$ contains at least two edges.

Lemma 2.5 Assume that $D$ is a van Kampen diagram, and $C \subset D$ is a simply connected cluster.

If $C$ is not complicated, then $\|\partial C\|_{\infty} \leq$ length $(\partial C),\|\partial C\|_{1} \leq 2$ length $(\partial C)$.

Proof Denote by $\widetilde{H}_{i}{ }^{\rho}$ the type of the cluster $C$, so that edges of $C$ are labeled by elements of $\widetilde{H}_{i}{ }^{\rho}$. If $C$ is not complicated, all edges of $\partial C$ but one have complexity 1. The cluster being simply connected, the label of the remaining edge has the same image in $\widetilde{H}_{i}{ }^{\rho}$ as a product of length $(\partial C)-1$ elements of $\left(\widetilde{S}_{i}{ }^{\rho}\right)^{ \pm 1}$. Therefore, this edge has complexity at most length $(\partial C)-1$. It follows that $\|\partial C\|_{\infty} \leq \operatorname{length}(\partial C)$, and $\|\partial C\|_{1} \leq($ length $(\partial C)-1)+\sum_{e \in \partial C} 1$. This proves the lemma.

Lemma 2.6 (See also [9, Lemma 2.27]) Let $D$ be a van Kampen diagram whose clusters are simply connected, noncomplicated and standardly filled.

Then $\left\|D_{\text {thick }}\right\|_{\infty} \leq 6 \operatorname{Area}(D)$.

Proof Any edge of $D_{\text {thick }}$ is either contained in a cell of type $\mathcal{R}^{\prime}$ (it has complexity 1 ) or in a cluster $C$. Since the number of edges of $D$ that lie in the boundary of a $2-$ cell is bounded by $3 \times \operatorname{Area}(D)$, we have length $(\partial C) \leq 3 \times \operatorname{Area}(D)$. Since $C$ is not complicated, $\|C\|_{\infty} \leq 6 \times \operatorname{Area}(D)$ by Lemma 2.5 . The lemma follows. 

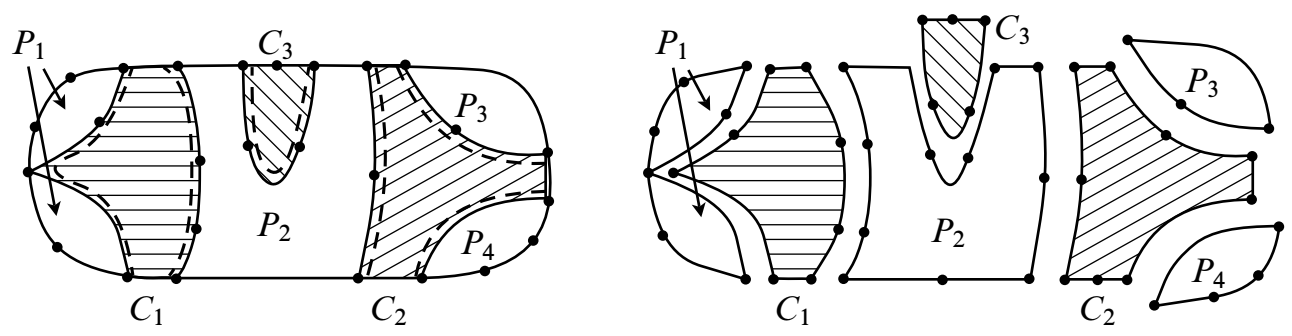

Figure 2: 3 complicated clusters, 4 regular pieces and 6 cluster arcs

\subsection{Cluster arcs and pieces}

In this section, we explain how to cut $D$ along the boundary components of the complicated clusters (we do not touch the noncomplicated clusters).

Consider a diagram $D$ whose clusters are simply connected. A cluster arc is a maximal subpath $c \subset \partial C$ for some complicated cluster $C$ that does not contain any edge of $\partial D$ (see Figure 2). Since $\partial C$ is an embedded circle, each cluster arc $c$ is an embedded arc with endpoints in $\partial D$, and $c \cap \partial D$ contains no edge, but it may contain vertices distinct from its endpoints.

We define regular pieces of $D$ as the connected components of $D \backslash \mathcal{C}$, where $\mathcal{C}$ denotes the interior in $D$ of the union of all complicated clusters in $D$ (edges in $\partial D \cap \partial C$ for some complicated cluster are in $\mathcal{C}$ ); see Figure 2. Regular pieces and complicated clusters are called pieces.

Here is an alternative description. For each complicated cluster $C$, consider properly embedded arcs with endpoints in $\partial D$, that are very close and parallel to each cluster arc, obtained by pushing inside $C$ the cluster arcs. Let $\mathcal{A}$ be the union of such embedded arcs when $C$ ranges over all complicated clusters. Then connected components of $D \backslash \mathcal{A}$ are in one-to-one correspondence with pieces. On Figure $2, \mathcal{A}$ is represented by dotted lines.

Although we won't need it, we note that this also makes sense if $D$ is not thick: edges of $D \backslash D_{\text {thick }}$ are contained in regular pieces.

Clearly, the set of pieces induces a partition of the set of 2-cells of $D$. There is a natural incidence graph $\mathcal{G}$ for this partition, whose vertices are the pieces, whose edges are the cluster arcs, the two endpoints of an edge being the cluster and the regular piece on both sides of the corresponding cluster arc.

Lemma 2.7 Let $D$ be a van Kampen diagram and assume that any cluster of $D$ is simply connected. The incidence graph $\mathcal{G}$ is a bipartite tree and the degree of a vertex 
$v$ associated to a complicated cluster $C$ is at most the number of edges in $\partial D \cap \partial C$, with strict inequality when $v$ is a leaf of the tree $\mathcal{G}$.

Proof The graph is bipartite by definition. It is connected because $D$ is connected. Since every cluster arc separates $D$, every edge of the incidence graph disconnects it. This proves that $\mathcal{G}$ is a tree.

Consider a vertex $v$ associated to a complicated cluster $C$. The degree of $v$ is, by definition, the number of cluster arcs on $\partial C$. Since $C$ is simply connected, $\partial C$ is an embedded circle, and since $C$ is complicated, $\partial C$ contains an edge of $\partial D$. By maximality in the definition of cluster arcs, each such arc is followed in $\partial C$ (with a chosen fixed orientation) by an edge of $\partial C \cap \partial D$. This association, which is clearly one-to-one, ensures the bound on the degree.

Finally, if $v$ is a leaf of $\mathcal{G}$, its degree is 1 and $\partial D \cap \partial C$ contains at least 2 edges because $C$ is complicated.

The following result of [3] was, to some extent, left to the reader. We include a proof.

Lemma 2.8 Let $D$ be a van Kampen diagram. If every cluster is simply connected, then the number of pieces, and the number of cluster arcs, are both bounded by length $(\partial D)$.

Proof The number $N$ of pieces is the number of vertices of the incidence graph $\mathcal{G}$. Since $\mathcal{G}$ is a tree, $N=E+1$, where $E$ is the number of edges of $\mathcal{G}$, ie the number of cluster arcs. Denote by $v_{C}$ the vertex corresponding to a cluster $C$, by $d\left(v_{C}\right)$ its degree, and by $V_{c l}$ the set of all vertices of $\mathcal{G}$ corresponding to clusters. Since $\mathcal{G}$ is bipartite, $E=\sum_{v_{C} \in V_{c l}} d\left(v_{C}\right)$. By Lemma 2.7, $d\left(v_{C}\right)$ is bounded by the number $e(C)$ of edges of $\partial C \cap \partial D$. Therefore $E \leq \sum_{v_{C} \in V_{c l}} e(C) \leq \operatorname{length}(\partial D)$.

Finally, if some $v_{C}$ is a leaf of $\mathcal{G}$, this last inequality is a strict inequality, which yields $N=E+1 \leq$ length $(\partial D)$. There remains the case where some leaf of $\mathcal{G}$ is a regular piece $B$. This means that $\partial B=\alpha \cup \beta$, where $\alpha$ is a cluster arc, and $\beta$ is a path in $\partial D$. Since clusters are simply connected, the endpoints of $\alpha$ are distinct, so $\beta$ contains at least an edge. This implies that $\sum_{v_{C} \in V_{c l}} e(C)<$ length $(\partial D)$, and concludes the proof.

\subsection{Reduction to diagrams of small complexity}

We are now ready to state and prove the main statement of this section. It claims that if $X_{\rho}$ does not satisfy a linear isoperimetric inequality, this shows up on diagrams of small area (this is Papasoglu's Theorem) and small complexity. 
Proposition 2.9 [3, Proposition 1.5] Let $K \geq 10^{6}$ and $\rho \in \mathbb{N} \cup\{\infty\}, \rho \geq 3 \times 240 K$. Assume that $X_{\rho}$ fails to satisfy a linear isoperimetric inequality of constant $K$ (that is, there exists a word $w$ over the alphabet $\widehat{S}_{\rho}$ such that $\operatorname{Area}(w)>K$ length $(w)$ ).

Then, there exists a word $w^{\prime \prime}$ over the alphabet $\widehat{S}_{\rho}$, and a minimal van Kampen diagram $D^{\prime \prime}\left(\right.$ over $\left.X_{\rho}\right)$ for $w^{\prime \prime}$, such that:

(1) $\operatorname{Area}\left(D^{\prime \prime}\right) \leq 240 K$

(2) $\left\|D^{\prime \prime}\right\|_{\infty} \leq 2.10^{6} K^{2}$

(3) $\operatorname{Area}\left(D^{\prime \prime}\right)>\frac{\sqrt{K}}{600}$ length $\left(\partial D^{\prime \prime}\right)$

Proof The first step is to apply Papasoglu's Theorem 1.1 to the presentation $X_{\rho}$ to obtain a word $w^{\prime}$ over $\widehat{S}_{\rho}$ for which

$$
K / 2 \leq \operatorname{Area}\left(w^{\prime}\right) \leq 240 K \text { and } \operatorname{Area}\left(w^{\prime}\right)>\frac{1}{2 \times 10^{4}} \operatorname{length}\left(w^{\prime}\right)^{2} .
$$

Using $\sqrt{\operatorname{Area}\left(w^{\prime}\right)}>$ length $\left(w^{\prime}\right) / \sqrt{2 \times 10^{4}}$ and $\operatorname{Area}\left(w^{\prime}\right) \geq K / 2$, we get

$$
\operatorname{Area}\left(w^{\prime}\right)>\sqrt{\frac{\operatorname{Area}\left(w^{\prime}\right)}{2 \times 10^{4}}} \times \operatorname{length}\left(w^{\prime}\right) \geq \frac{\sqrt{K}}{200} \times \operatorname{length}\left(w^{\prime}\right) .
$$

Choose a diagram $D^{\prime}$ among minimal area diagrams over $X_{\rho}$ for $w^{\prime}$ so that the number of 2 -cells of type $\mathcal{R}^{\prime}$ is minimal. We claim that up to changing $w^{\prime}$, we can assume that $D^{\prime}$ is thick, ie all edges lie in the boundary of a 2-cell. Indeed, if all connected components $A_{1}^{\prime}, \ldots, A_{l}^{\prime}$ of $D_{\text {thick }}^{\prime}$ satisfy $\operatorname{Area}\left(A_{i}^{\prime}\right) \leq(\sqrt{K} / 200) \times \operatorname{length}\left(\partial A_{i}^{\prime}\right)$, then

$$
\operatorname{Area}\left(D^{\prime}\right)=\sum_{i} \operatorname{Area}\left(A_{i}^{\prime}\right) \leq \frac{\sqrt{K}}{200} \sum_{i} \operatorname{length}\left(\partial A_{i}^{\prime}\right) \leq \frac{\sqrt{K}}{200} \times \operatorname{length}\left(w^{\prime}\right),
$$

which is a contradiction. It follows that some component $A_{i}^{\prime}$ satisfies $\operatorname{Area}\left(A_{i}^{\prime}\right)>$ $(\sqrt{K} / 200) \times \operatorname{length}\left(\partial A_{i}^{\prime}\right)$. Obviously, $\operatorname{Area}\left(A_{i}^{\prime}\right) \leq \operatorname{Area}\left(D^{\prime}\right) \leq 240 K$, and $A_{i}^{\prime}$ is a diagram for $\partial A_{i}^{\prime}$ that minimizes the area and the number of cells of type $\mathcal{R}^{\prime}$ (if not, substituting a diagram of smaller area for $\partial A_{i}^{\prime}$ in $D^{\prime}$ contradicts minimality of $D^{\prime}$ ). This proves that we can assume that $D^{\prime}$ is thick.

We do not have any control on the complexity of a diagram filling $w^{\prime}$ yet. Since $\rho \geq 3 \times 240 K$, Lemma 2.4 shows that the clusters of $D^{\prime}$ are simply connected. We can modify $D^{\prime}$ and assume that all clusters are standardly filled. By Remark $2.3, D^{\prime}$ still minimizes area and the number of cells of type $\mathcal{R}^{\prime}$. By Lemma 2.8, the number of pieces in the decomposition into complicated clusters and regular pieces is at most length $\left(\partial D^{\prime}\right)$. 

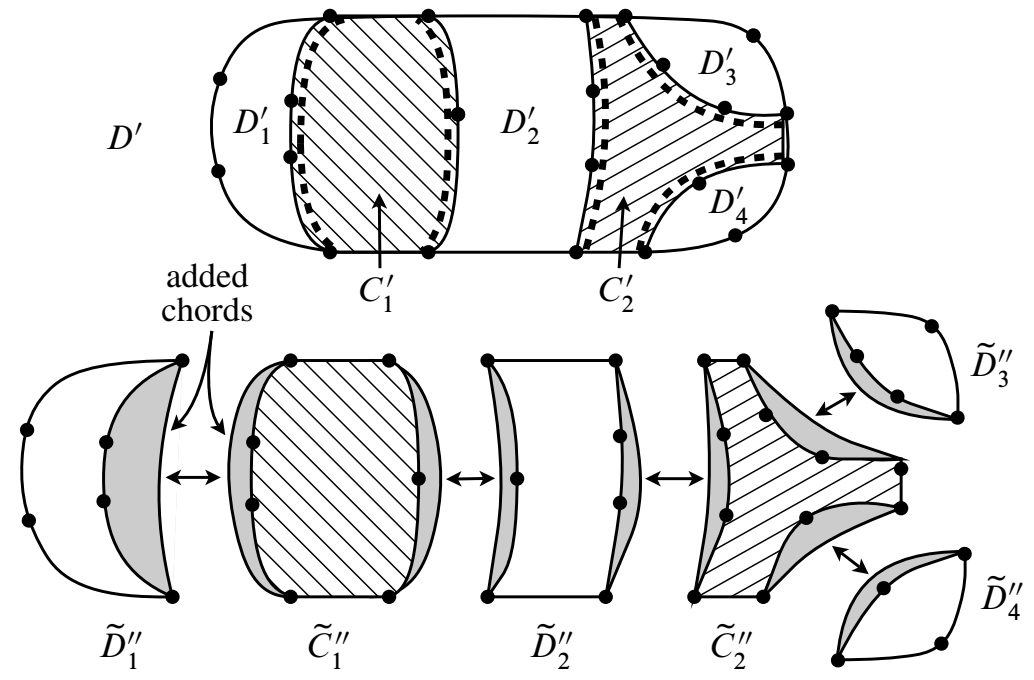

Figure 3: Adding chords to the pieces of $D^{\prime}$, and regluing them together

Let $C_{1}^{\prime}, \ldots, C_{s}^{\prime}$ be the complicated clusters of $D^{\prime}$, and $D_{1}^{\prime}, \ldots, D_{r}^{\prime}$, be the regular pieces. We construct new diagrams $C_{i}^{\prime \prime}, D_{j}^{\prime \prime}$, and $\widetilde{C}_{i}^{\prime \prime}, \widetilde{D}_{j}^{\prime \prime}$ from $C_{i}^{\prime}, D_{j}^{\prime}$ by first adding chords, then by changing the triangulation as follows (see Figure 3).

Fix a complicated cluster $C_{k}^{\prime}$ of $D^{\prime}$, and denote by $\widetilde{H}_{i}{ }^{\rho}$ its type. Its boundary $\partial C_{k}^{\prime}$ is a union of pairwise disjoint cluster arcs, together with arcs in $\partial D^{\prime}$. Consider a cluster arc $c \subset \partial C_{k}^{\prime}$ whose edges are labeled by elements $a_{1}, \ldots, a_{n}$ of $\widetilde{H}_{i}{ }^{\rho}$, and let $a_{c}=a_{1} \cdots a_{n} \in \widetilde{H}_{i}{ }^{\rho}$ be their product. We glue along $c$ a standardly filled disk with boundary labeled by $a_{1}, \ldots, a_{n}, a_{c}^{-1}$. We name the new edge labeled by $a_{c}^{-1}$ a chord. Performing this operation for each cluster arc, we get a disk $C_{k}^{\prime \prime}$ made of cells of type $\widetilde{H}_{i}{ }^{\rho}$. Finally, we change the triangulation of this disk to a standard filling, and we call $\widetilde{C}_{k}^{\prime \prime}$ the obtained diagram. Note that $\operatorname{Area}\left(\widetilde{C}_{k}^{\prime \prime}\right) \leq \operatorname{length}\left(\partial \widetilde{C}_{k}^{\prime \prime}\right)-2$.

Now, we perform a similar operation for each regular piece $D_{j}^{\prime}$. For each cluster arc $c \subset \partial D_{j}^{\prime}$ labeled by $a_{1}, \ldots, a_{n} \in \widetilde{H}_{i}{ }^{\rho}$ (now, the type $\widetilde{H}_{i}{ }^{\rho}$ may depend on $c$ ), we define $a_{c}=a_{1} \ldots a_{n} \in \widetilde{H}_{i}{ }^{\rho}$, and glue to $C_{k}^{\prime}$ along $c$ a new cluster of type $\widetilde{H}_{i}{ }^{\rho}$, standardly filled, whose boundary is labeled by $a_{1}, \ldots, a_{n}, a_{c}^{-1}$. Since the filling is standard, the area of the added cluster is $(n+1)-2=\operatorname{length}(c)-1$. Performing this operation for each cluster arc, we get the new diagram $D_{j}^{\prime \prime}$. Finally, we take for $\widetilde{D}_{j}^{\prime \prime}$ a diagram with boundary $\partial D_{j}^{\prime \prime}$, and minimizing successively the area and the number of 2-cells of type $\mathcal{R}^{\prime}$.

We are going to bound $\left\|\widetilde{D}_{j}^{\prime \prime}\right\|_{\infty}$ by first bounding $\left\|D_{j}^{\prime \prime}\right\|_{\infty}$. Since all complicated clusters of $D^{\prime}$ are $C_{1}^{\prime}, \ldots, C_{s}^{\prime}, D_{j}^{\prime \prime}$ has no complicated cluster coming from $D^{\prime}$. The 
newly created clusters in $D_{j}^{\prime \prime}$ have just one edge in $\partial D_{j}^{\prime \prime}$, so are not complicated. Therefore, clusters of $D_{j}^{\prime \prime}$ are not complicated, simply connected and standardly filled. Since $D^{\prime}$ is thick, so is $D_{j}^{\prime \prime}$. Applying Lemma 2.6 to $D_{j}^{\prime \prime}$, we get

$$
\left\|D_{j}^{\prime \prime}\right\|_{\infty} \leq 6 \times \operatorname{Area}\left(D_{j}^{\prime \prime}\right) \leq 6 \times 240 K .
$$

In particular, $\left\|\partial \widetilde{D}_{j}^{\prime \prime}\right\|_{\infty}=\left\|\partial D_{j}^{\prime \prime}\right\|_{\infty} \leq 6 \times 240 K$, and since $D_{j}^{\prime \prime}$ is thick, $\left\|\partial D_{j}^{\prime \prime}\right\|_{1} \leq$ $3 \operatorname{Area}\left(D_{j}^{\prime \prime}\right)\left\|\partial D_{j}^{\prime \prime}\right\|_{\infty} \leq 18 \times(240 K)^{2}$. Applying Lemma 2.4 to $\widetilde{D}_{j}^{\prime \prime}$, we get

$$
\left\|\widetilde{D}_{j}^{\prime \prime}\right\|_{\infty} \leq 3 \operatorname{Area}\left(D_{j}^{\prime \prime}\right)+\left\|\partial D_{j}^{\prime \prime}\right\|_{1} \leq 3 \times 240 K+18 \times(240 K)^{2} \leq 2.10^{6} K^{2} .
$$

This proves that for all $j \in\{1, \ldots, r\}, \tilde{D}_{j}^{\prime \prime}$ satisfies assertions (1) and (2) of the proposition.

We now prove that one of the diagrams $\widetilde{D}_{j}^{\prime \prime}, j=1, \ldots, r$ must satisfy (3). Assume by contradiction that for all $j \in\{1, \ldots, r\}$, Area $\left(\widetilde{D}_{j}^{\prime \prime}\right) \leq(\sqrt{K} / 600) \operatorname{length}\left(\widetilde{D}_{\widetilde{D}}^{\prime \prime}\right)$. Note that $\widetilde{C}_{k}^{\prime \prime}$ satisfies this inequality as well. Indeed, $\operatorname{Area}\left(\widetilde{C}_{k}^{\prime \prime}\right) \leq$ length $\left(\partial \widetilde{C}_{k}^{\prime \prime}\right)$, and by assumption, $K \geq 10^{6}$ so $(\sqrt{K} / 600) \geq 1$.

Gluing together the diagrams $\widetilde{D}_{1}^{\prime \prime}, \ldots, \widetilde{D}_{r}^{\prime \prime}$ and $\widetilde{C}_{1}^{\prime \prime}, \ldots, \widetilde{C}_{s}^{\prime \prime}$ pairwise along the two chords corresponding to a given cluster arc as shown on Figure 3, we get another (not necessarily minimal) van Kampen diagram $\widetilde{D}^{\prime}$ for $w^{\prime}$.

We have

$$
\begin{aligned}
\operatorname{Area}\left(D^{\prime}\right) \leq \operatorname{Area}\left(\widetilde{D}^{\prime}\right) & =\sum_{j} \operatorname{Area}\left(\widetilde{D}_{j}^{\prime \prime}\right)+\sum_{k} \operatorname{Area}\left(\widetilde{C}_{k}^{\prime \prime}\right) \\
& \leq \frac{\sqrt{K}}{600}\left(\sum_{j} \operatorname{length}\left(\partial \widetilde{D}_{j}^{\prime \prime}\right)+\sum_{k} \operatorname{length}\left(\partial \widetilde{C}_{k}^{\prime \prime}\right)\right) \\
& \leq \frac{\sqrt{K}}{600}\left(\operatorname{length}\left(\partial D^{\prime}\right)+2 n_{a}\right),
\end{aligned}
$$

where $n_{a}$ is the number of cluster arcs in $D^{\prime}$. By Lemma $2.8, n_{a} \leq$ length $\left(\partial D^{\prime}\right)$, so $\operatorname{Area}\left(D^{\prime}\right) \leq(\sqrt{K} / 200) \times$ length $\left(\partial D^{\prime}\right)$, thus contradicting the property of $D^{\prime}$ established at the beginning of the proof.

\section{Consequences}

Corollary 3.1 Assume that $X_{\infty}$ satisfies a linear isoperimetric inequality of factor $K \geq 10^{6}$. Let $K^{\prime}=(600 K)^{2}$ and $\rho_{0}(K)=10^{26} K^{5}$ Then for all $\rho \geq \rho_{0}(K), X_{\rho}$ satisfies a linear isoperimetric inequality of factor $K^{\prime}$. 
Before proving the corollary, we need to relate more explicitly the presentations $X_{\rho}$ and $X_{\infty}$. Recall that $\hat{S}_{\rho}=S \sqcup \widetilde{H}_{1}{ }^{\rho} \sqcup \cdots \sqcup \tilde{H}_{n}^{\rho}$ and $\hat{S}_{\infty}=S \sqcup \widetilde{H}_{1} \sqcup \cdots \sqcup \widetilde{H}_{n}$ the corresponding generating sets, and that we have morphisms $p_{i}{ }^{\rho}: \widetilde{H}_{i}{ }^{\rho} \rightarrow H_{i}$ and isomorphisms $p_{i}: \widetilde{H}_{i} \rightarrow H_{i}$. The morphisms

$$
q_{i}=p_{i}^{-1} \circ p_{i}^{\rho}: \widetilde{H}_{i}^{\rho} \rightarrow \widetilde{H}_{i}
$$

induce an obvious map $p: \hat{S}_{\rho} \rightarrow \hat{S}_{\infty}$ that is the identity on $S$ and maps $\tilde{H}_{i}{ }^{\rho}$ to $\tilde{H}_{i}$ through $p_{i}^{-1} \circ p_{i}{ }^{\rho}$. If $w=a_{1} \cdots a_{n}$ is a word over $\widehat{S}_{\rho}$, we denote by $p(w)=$ $p\left(a_{1}\right) \cdots p\left(a_{n}\right)$ the corresponding word over $\hat{S}_{\infty}$. Clearly, if $w$ is any relator of the presentation $X_{\rho}$, then $p(w)$ is a relator of $X_{\infty}$. It follows that given any diagram $D$ over $X_{\rho}$ for a word $w$, one gets a new diagram $p_{*}(D)$ for $p(w)$ over $X_{\infty}$ by applying the map $p$ to all the labels of all edges of $D$.

On the other hand, $q_{i}$ induces a bijection between the balls of radius $\rho / 2$ of $\widetilde{H}_{i}{ }^{\rho}$ and $\widetilde{H}_{i}$, whose inverse we denote by $q_{i}^{-1}$. Similarly, we denote by $p^{-1}$ the inverse of the restriction of $p: \hat{S}_{\rho} \rightarrow \widehat{S}_{\infty}$ to the set of elements of complexity at most $\rho / 2$. Now, if $a, b, c \in H_{i}$ are in the ball of radius $\rho / 3$ of $H_{i}$ and satisfy $a b c=1$ in $\widetilde{H}_{i}$, then $q_{i}^{-1}(a) q_{i}^{-1}(b) q_{i}^{-1}(c)=1$ in $\tilde{H}_{i}{ }^{\rho}$. This means that if some diagram $D$ over $X_{\infty}$ for $w$ satisfies $\|D\|_{\infty} \leq \rho / 3$, then the diagram $p_{*}^{-1}(D)$ (with obvious notations) is a diagram over $X_{\rho}$ for $p^{-1}(w)$.

Proof of Corollary 3.1 Assume that $X_{\rho}$ fails to satisfy the predicted isoperimetric inequality (of factor $K^{\prime}$ ), and argue towards a contradiction. By Proposition 2.9, there is a word $w^{\prime \prime}$ representing the trivial element, with a diagram $D^{\prime \prime}$, minimal over the presentation $X_{\rho}$, of area at most $240 K^{\prime}$, and complexity $\left\|D^{\prime \prime}\right\|_{\infty} \leq 2.10^{6} K^{\prime 2}$ and such that $\operatorname{Area}\left(D^{\prime \prime}\right)>\left(\sqrt{K^{\prime}} / 600\right) \times \operatorname{length}\left(w^{\prime \prime}\right)=K \times \operatorname{length}\left(w^{\prime \prime}\right)$.

Consider the map $p: \hat{S}_{\rho} \rightarrow \widehat{S}_{\infty}$ described above. Choose $D_{0}^{\prime \prime}$ among diagrams for $p\left(w^{\prime \prime}\right)$ in the presentation $X_{\infty}$, in order to minimize successively the area, the number of 2-cells of type $\mathcal{R}^{\prime}$, and the complexity $\left\|D_{0}^{\prime \prime}\right\|_{\infty}$. Since $X_{\infty}$ satisfies a linear isoperimetric inequality of factor $K, \operatorname{Area}\left(D_{0}^{\prime \prime}\right) \leq K \times \operatorname{length}\left(w^{\prime \prime}\right)$. It follows from the estimate of the previous paragraph, that $\operatorname{Area}\left(D_{0}^{\prime \prime}\right)<\operatorname{Area}\left(D^{\prime \prime}\right)$ which is itself $\leq 240 K^{\prime}$.

By Lemma 2.4, $\left\|D_{0}^{\prime \prime}\right\|_{\infty} \leq 720 K^{\prime}+\left\|p\left(w^{\prime \prime}\right)\right\|_{1}$. Let us estimate the terms:

$$
\begin{aligned}
\left\|p\left(w^{\prime \prime}\right)\right\|_{1} \leq\left\|w^{\prime \prime}\right\|_{1} \leq \text { length }\left(w^{\prime \prime}\right)\left\|D^{\prime \prime}\right\|_{\infty} & \leq \text { length }\left(w^{\prime \prime}\right) \times 2.10^{6} K^{\prime 2} \\
& \leq \frac{1}{K} \operatorname{Area}\left(D^{\prime \prime}\right) \times 2.10^{6} K^{\prime 2} \\
& \leq \frac{1}{K} \times 240 K^{\prime} \times 2.10^{6} K^{\prime 2} \leq 3.10^{25} K^{5} .
\end{aligned}
$$


Also, since $K \geq 10^{6}$, one has $720 K^{\prime} \leq 10^{9} K^{2} \leq K^{5}$. By hypothesis on $\rho$, we see that $\left\|D_{0}^{\prime \prime}\right\|_{\infty} \leq \rho / 3$. It follows that $p_{*}^{-1}\left(D_{0}^{\prime \prime}\right)$ is a diagram over $X_{\rho}$ for $w^{\prime \prime}$. We already noticed that it has area $<\operatorname{Area}\left(D^{\prime \prime}\right)$, a contradiction to the minimality of $D^{\prime \prime}$ over the presentation $X_{\rho}$.

Lemma 3.2 Assume that $X_{\rho}$ satisfies a linear isoperimetric inequality of factor $K^{\prime}$ with $\rho \geq \max \left(3 K^{\prime}, 2\right)$.

Then $p_{i}{ }^{\rho}: \widetilde{H}_{i}{ }^{\rho} \rightarrow H_{i}$ is an isomorphism. In particular, $H_{i}$ is finitely presented, with a presentation whose defining relations are of length $\leq \rho$.

Proof Assume for contradiction that $p_{i}: \widetilde{H}_{i}{ }^{\rho} \rightarrow H_{i}$ is not injective, and consider $a \in \operatorname{ker} p_{i} \backslash\{1\}$. Then $a$ is a generator of the presentation $X_{\rho}$ that represents the trivial element of $G$. Note that since $\rho>1, a \notin \widetilde{S}_{i}{ }^{\rho}$. Therefore, there exists a van Kampen diagram $D$ over $X_{\rho}$ whose boundary consists of a single edge $e$ labeled $a$, and whose area is at most $K^{\prime}$. We choose a diagram for $a$ over $X_{\rho}$ in order to minimize successively the area, the number of 2 -cells of type $\mathcal{R}^{\prime}$ and $\|D\|_{\infty}$. Since $\rho \geq 3 K^{\prime}$, Lemma 2.4 implies that clusters of $D$ are simply connected. Since $a \notin \widetilde{S}_{i}{ }^{\rho}, e$ lies in a cluster $C$ of type $\widetilde{H}_{i}{ }^{\rho}$. But since $C$ is simply connected, and since a cluster of type $\widetilde{H}_{i}^{\rho}$ involves only relations of $\widetilde{H}_{i}{ }^{\rho}$, we get that $a$ is trivial in $\widetilde{H}_{i}{ }^{\rho}$, a contradiction.

Corollary 3.3 Assume that $X_{\infty}$ satisfies a linear isoperimetric inequality of factor $K$. Let $\rho_{0}$ be the function defined in Corollary 3.1.

Then the subgroups $H_{i}$ are finitely presented, with a presentation whose defining relations are of length $\leq \rho_{0}\left(\max \left(K, 10^{6}\right)\right)$.

Proof Without loss of generality, we can assume $K \geq 10^{6}$. By Corollary 3.1, $X_{\rho_{0}(K)}$ satisfies a linear isoperimetric inequality of factor $K^{\prime}=(600 K)^{2}$. Lemma 3.2 concludes.

Lemma 3.4 (See also [9, Lemma 5.4]) Assume that $X_{\infty}$ satisfies a linear isoperimetric inequality of factor $K$.

If $s \in S$ represents an element $a$ of $\tilde{H}_{i}$, then $\|a\| \leq 12 K$.

Proof The word $w=s^{-1} a$ is a word of length 2 over $X_{\infty}$. If it represents the trivial element in $G$, then there is a van Kampen diagram $D$ over $X_{\infty}$ whose boundary is a path of length 2 labeled $s^{-1} a$, and whose area is at most $2 K$. We choose $D$ among minimal area diagrams over $X_{\infty}$ for $w$ so that the number of 2-cells of type $\mathcal{R}^{\prime}$ is 
minimal. Lemma 2.4 (applied with $\rho=\infty$ ) implies that clusters of $D$ are simply connected, and we can assume that they are standardly filled.

Note that there is no complicated cluster as only the edge labeled $a$ of $\partial D$ can be in a cluster. By Lemma 2.6, this implies that $\left\|D_{\text {thick }}\right\|_{\infty} \leq 12 K$, so $\|a\| \leq 12 K$.

We obtain the following improvement of [3]:

Corollary 3.5 There exists an algorithm that takes as input a finite presentation of a group $G$, a solution of its word problem and a collection of finite subsets $S_{1}, \ldots, S_{n} \subset$ $G$, and that terminates if and only if $G$ is hyperbolic relative to $\left\langle S_{1}\right\rangle, \ldots,\left\langle S_{n}\right\rangle$.

In this case, produces an isoperimetry constant $K$ for the presentation $X_{\infty}$, a finite presentation for each of the parabolic subgroups, and says whether $G$ is parabolic (ie $G=\left\langle S_{i}\right\rangle$ for some $i$ ).

Proof For a fixed $K \geq 10^{6}$, we consider all diagrams $D$ over $X_{\infty}$ such that $\|D\|_{\infty} \leq$ $B=2.10^{6} K^{2}$ and Area $(D) \leq 240 K$. There are only finitely many. The word problem in $G$ allows to list all relators of $\left\langle S_{i}\right\rangle$ of length at most $3 B$, to compute the ball of radius $3 B$ in the Cayley graph of $H_{i}$ with respect to the generating set $S_{i}$ and hence to list all these diagrams. Out of this list, we make the list $\mathcal{W}(K)$ of words labeling the boundaries of these diagrams.

We claim that given $w \in \mathcal{W}(K)$, we can compute $\operatorname{Area}(w)$. Indeed, let $D^{\prime}$ be a diagram for $w$ chosen to minimize area, the number of cells of type $\mathcal{R}^{\prime}$, and $\left\|D^{\prime}\right\|_{\infty}$. By Lemma 2.4, $\left\|D^{\prime}\right\|_{\infty} \leq 3 \operatorname{Area}\left(D^{\prime}\right)+\|w\|_{1} \leq 720 K+\|w\|_{1}$. We can compute the upper bound $M=720 K+\|w\|_{1}$ for $\left\|D^{\prime}\right\|_{\infty}$, and we can list all diagrams $D^{\prime}$ with $\operatorname{Area}\left(D^{\prime}\right) \leq 240 K$ and $\left\|D^{\prime}\right\|_{\infty} \leq M$ whose boundary is $w$. We can then compute $\operatorname{Area}(w)$ as the minimal area of these diagrams.

Now we can check whether $\operatorname{Area}(w) \leq(\sqrt{K} / 600)$ length $(w)$ for all $w \in \mathcal{W}(K)$. If this is not the case, the algorithm increments $K$ and starts over.

If this is the case, then by Proposition $2.9, X_{\infty}$ satisfies isoperimetric inequality of factor $K$, and the algorithm stops. It outputs $K$, and gives as set of relators for $\left\langle S_{i}\right\rangle$, the set of all words of length $\leq \rho_{0}(K)$ that are trivial in $G$; this can be done using the word problem in $G$, and this is indeed a presentation of $\left\langle S_{i}\right\rangle$ by Corollary 3.3. To check whether $G=\left\langle S_{i}\right\rangle$, one needs to check whether each $s \in S$ represents an element $a \in\left\langle S_{i}\right\rangle$. Lemma 3.4 bounds the complexity of $a$, and we can try all possibilities for $a$ using the word problem.

If $X_{\infty}$ does satisfy a linear isoperimetric inequality of factor $K_{0}$, then the process will obviously stop when $K$ will reach a value greater than $\left(600 K_{0}\right)^{2}$. 
Corollary 3.6 There exists an algorithm as follows. It takes as input a finite presentation of a group $G$, a solution for its word problem and a recursive class of finitely presented groups $\mathcal{C}$ (given by a Turing machine enumerating them). It terminates if and only if $G$ is properly hyperbolic relative to subgroups that are in the class $\mathcal{C}$.

In this case, the algorithm produces an isoperimetry constant $K$, a generating set and a finite presentation for each of the parabolic subgroups.

Proof First, enumerate all possible presentations of groups in $\mathcal{C}$ using the Turing machine given as input, and Tietze transformations. Denote by $L$ the set of currently proposed presentations. In a second parallel process, list all possible families of finite subsets $\mathcal{S}=\left(S_{1}, \ldots, S_{n}\right)$ of $G$. For each of them, run in parallel the algorithm of Corollary 3.5 that stops if $G$ is hyperbolic relative to $\left\langle S_{1}\right\rangle, \ldots,\left\langle S_{n}\right\rangle$ and outputs a presentation of $\left\langle S_{i}\right\rangle$ in this case, and says whether $G$ is parabolic. For each $\mathcal{S}$ such that $G$ is hyperbolic relative to $\left\langle S_{1}\right\rangle, \ldots,\left\langle S_{n}\right\rangle$ and is not parabolic, record the obtained tuple of presentations $P_{\mathcal{S}}=\left(\mathcal{P}_{1}, \ldots, \mathcal{P}_{n}\right)$.

We thus have two enumerations: one of the presentations of the groups in $\mathcal{C}$ (the list $L$ ), and one of the proper relatively hyperbolic structures of $G$, with presentations of the parabolic subgroups. At each step compare the set of recorded tuples $P_{\mathcal{S}}$ with the presentations in the list $L$. If for some $\mathcal{S}$ all the presentations of the tuple $P_{\mathcal{S}}$ are listed in $L$, then stop.

\section{A geometric proof of the finite presentation}

After listening to a talk by V Gerasimov, we realized that Theorem 1 about the finite presentation of parabolic subgroups can be proved using the following geometric argument which is developed further in Gerasimov and Potyagailo's [4].

We recall a construction of a proper hyperbolic space $X$ for $G$ (see Bowditch [2], Groves and Manning [6]; see also Hruska [7, Theorem 4.4]). Let $G$ be relatively hyperbolic relative to finitely generated subgroups $H_{1}, \ldots, H_{n}$. We take a finite generating set $S_{i}$ for each $H_{i}$ and we take $Y$ a Cayley 2-complex of $G$ for a generating set $S$ containing each $S_{i}$. We denote by $C_{i}$ the Cayley graph of $H_{i}$ with respect to $S_{i}$, which we view as a subgraph of $Y$. For each $i$, consider a combinatorial horoball based on $C_{i}$ : this is a graph with vertices $V\left(C_{i}\right) \times \mathbb{N}$, where there is an edge between $(x, i)$ and $(x, i+1)$, and an edge between $(x, i)$ and $(y, i)$ if $d_{C_{i}}(x, y) \leq 2^{i}$. We view $C_{i}$ as the subset $C_{i} \times\{0\}$ of $B_{i}$. Then we define $X$ by gluing on $Y$ a copy of $B_{i}$ on each $g C_{i}$ for each $g \in G / H_{i}$. If $G$ is hyperbolic relative to $H_{1}, \ldots, H_{n}$, then $X$ is a Gromov-hyperbolic space [6]. 
Let $p: X \rightarrow B_{i}$ be the closest point projection for the metric in $X$. Clearly, $p(Y) \subset C_{i}$. Quasiconvexity of $B_{i}$ shows that $p$ is coarsely Lipshitz. The projection of each 2-cell of $Y$ is a uniformly bounded subset of $C_{i}$ (for the metric on $X$, hence for its intrinsic metric). Let $K$ be a bound on the diameter in $C_{i}$ of the projection of a 2-cell of $Y$, and let $C_{i}^{\prime}$ be the corresponding Rips complex of $C_{i}$, where one adds a simplex on a set of vertices $S \subset C_{i}$ whenever $\operatorname{diam}(S) \leq K$.

Let $c$ be any cycle in $C_{i}$. Thus $c$ can be viewed as a path in $Y$. Since $G$ is finitely presented, $c$ bounds a disk $D$ in $Y$. Then the projection of each 2-cell of $Y$ can be filled by a $2-$ cell in $C_{i}^{\prime}$, so $c$ is nullhomotopic in $C_{i}^{\prime}$. Since this argument is independent of the choice of the path $c$, this proves that $C_{i}^{\prime}$ is simply connected. Since $C_{i}^{\prime} / H_{i}$ is compact, it follows that $H_{i}$ is finitely presented.

This argument can be refined to show that if $G$ has a finite classifying space (resp. is of type $F P_{n}$ ), then so are its parabolic subgroups.

\section{References}

[1] B H Bowditch, Notes on Gromov's hyperbolicity criterion for path-metric spaces, from: "Group theory from a geometrical viewpoint", (É Ghys, A Haefliger, A Verjovsky, editors), World Sci. Publ., River Edge, NJ (1991) 64-167 MR1170364

[2] B H Bowditch, Relatively hyperbolic groups, Internat. J. Algebra Comput. 22 (2012) MR2922380

[3] F Dahmani, Finding relative hyperbolic structures, Bull. Lond. Math. Soc. 40 (2008) 395-404 MR2418795

[4] V Gerasimov, L Potyagailo, Quasiconvexity in the relatively hyperbolic groups arXiv:1103.1211

[5] M Gromov, Hyperbolic groups, from: "Essays in group theory", (S M Gersten, editor), Math. Sci. Res. Inst. Publ. 8, Springer, New York (1987) 75-263 MR919829

[6] D Groves, J F Manning, Dehn filling in relatively hyperbolic groups, Israel J. Math. 168 (2008) 317-429 MR2448064

[7] G C Hruska, Relative hyperbolicity and relative quasiconvexity for countable groups, Algebr. Geom. Topol. 10 (2010) 1807-1856 MR2684983

[8] R C Lyndon, P E Schupp, Combinatorial group theory, Classics in Mathematics 14, Springer, Berlin (2001) MR1812024

[9] D V Osin, Relatively hyperbolic groups: intrinsic geometry, algebraic properties, and algorithmic problems, Mem. Amer. Math. Soc. 179 (2006) 100 MR2182268 
[10] P Papasoglu, An algorithm detecting hyperbolicity, from: "Geometric and computational perspectives on infinite groups", (G Baumslag, D Epstein, R Gilman, H Short, C Sims, editors), DIMACS Ser. Discrete Math. Theoret. Comput. Sci. 25, Amer. Math. Soc. (1996) 193-200 MR1364185

Institut Fourier, Université de Grenoble 1

100 rue des Maths, BP 76, 38402 St. Martin d'Hères Cedex, France

Institut de recherche en mathématiques de Rennes, Université de Rennes 1

263 avenue du Général Leclerc, CS 74205, 35042 Rennes Cedex, France

francois.dahmani@ujf-grenoble.fr, vincent.guirardel@univ-rennes1.fr

http://www-fourier.ujf-grenoble.fr/ dahmani/,

http://perso.univ-rennes1.fr/vincent.guirardel/

Received: 3 October $2011 \quad$ Revised: 5 October 2012 